\title{
Application of Pattern Recognition for a Welding Process
}

\author{
Oto Haffner, Erik Kučera, Štefan Kozák, Erich Stark \\ Faculty of Electrical Engineering and Information Technology \\ Slovak University of Technology in Bratislava \\ Bratislava, Slovakia \\ Email: oto.haffner@stuba.sk
}

\begin{abstract}
The paper deals with the development of a system for automatic weld recognition using new information technologies based on cloud computing and single-board computer in the context of Industry 4.0. The proposed system is based on a visual system for weld recognition, and a neural network based on cloud computing for real-time weld evaluation, both implemented on a single-board low-cost computer. The proposed system was successfully verified on welding samples which correspond to a real welding process in the car production process. The system considerably contributes to the welds diagnostics in industrial processes of small- and medium-sized enterprises.
\end{abstract}

\section{INTRODUCTION}

$\mathrm{T}$ HE quality of manufacturing process is one of the most important aspect of production. Welding processes are used in many manufacturing processes and automotive industry. This makes prerequisites for researching and developing new modern methods and system for welding quality evaluation.

Visual inspection of the weld is one of the weld quality diagnostic methods for weld quality diagnostic. This method enables to check weld joint and its various parameters. Visual inspection does not destruct the weld so it is notdestructive methods for weld joints and materials. This examination is done as the first examination and it detects various defects. There is no need to use another method after detecting a defect with this method, so it is cost and timesaving.

This work deals with application for evaluation algorithm and weld recognition. It is based on the single-board computer using the visual system. This method is based on the method for visual quality evaluation and modern computer vision and image processing methods. Industry production, as well as mobile robotics, will find the usage of these methods.

This work has been supported by the Cultural and Educational Grant Agency of the Ministry of Education, Science, Research and Sport of the Slovak Republic, KEGA 030STU-4/2015 and KEGA 030STU-4/2017, by the Scientific Grant Agency of the Ministry of Education, Science and by the Slovak Research and Development Agency APVV-0772-12.
Actual trend in the industry is Industry 4.0 also named fourth industrial revolution. It is collective name for current automatization, exchanging of data and manufacturing technologies. It can be defined as a collective name for technologies and concept for organising value chain, which unites Cyber-Physical Systems, Internet of Thing and Internet of Service [1]. Industry 4.0 understands manufacturing units as complex distributed systems made by "smart" partial integration of individual autonomous subsystems. The integration is provided by suitable communication of each other based on actual demand, activity coordination and coordination among autonomous subsystems. [8]

Authors [7] uses improved Beamlet Transformation for weld edge detection. Images they work with are loaded by noise. The aim of authors work is to detect edge borders of welds. Beamlet algorithm does the dynamic thresholding in one of the steps. The algorithm predicts the directional characteristic of the weld and it is possible to filtrate unsuitable edges. Using this method, it is capable of directly extracting weld seam edges from highly noisy welding images without any pre-processing or post-processing steps, thus is characterized by its high efficiency and its prominent anti-noising performance.

Authors [11] worked with weld images with very low contrast. The weld images come from the pipeline and are loaded with low contrast and noise which do problems to convent edge detectors. At first, the image is noise filtered using morphological operation of opening and closing. The next is improved algorithm of fuzzy edge detection. The algorithm is based on two steps: multi-level fuzzy image improvement based on interactive searching of optimal threshold level and based on multi-directional edge detector, which convolution kernel is $5 \times 5$ with 8 directions and is based on gradient searching. The result of the algorithm was compared with detectors as Sobel, canny FED and fast FED. Authors obtained whit their method better results.

Edge detection and histogram projection use authors [9] They compare histogram projections of tested welds with set similarity threshold. This threshold evaluates the quality of the weld. Loaded image of the pattern has the same 
specifications- width and position as the tested image. One vertical line is tested from the pattern and tested image. The correlation threshold is set. Line histogram od pattern image and the tested image is computed. The correlation degree of two histograms using Tukey HSD difference is computed. If the degree is lower than set threshold, this part of the image contains edge defects. The procedure is repeated over the entire width.

Authors [10] deals with weld evaluation on metal cans. On the weld can be a defect wich do not directly relate to weld (rest of glue, dust etc.). Because of this, authors use probability evaluation of two evaluation methods. The first, Column Gray-Level Accumulation Inspection represents in general histogram projection. The histogram projections of pattern and rested weld are compared. They compare also the first derivation for making better results. Defects of the wider surface can be detected by this method. DempsterShafer theory of evidence for overall evaluation is used.

Authors in other work [12] deal with edge detection based on pixel intensity difference of foreground and background. The background pixels intensity is occurred with maximal probability and the distribution of background pixels fits Gauss distribution.

\section{PROBLEM FORMULATION}

One of the most important production process for a wide range of products is also welding. Visual inspection of welds is one of the most effective and cheap unrestrictive methods for weld joints. This method of inspection is made by a manhuman vision. However, this can be loaded by human error because of fatigue, stress, inattention or lack of experience. In some manufactures, weld view is provided by the camera system. This image is shown on the monitor and the operator based on this image determine if the weld fits the set criteria. In the cases like this, it is possible to use automated method for weld evaluation by the visual system.

Incoming new modern trend Industry 4.0 and Internet of Things request new needs for all parts of manufacturing systems. It requests capability to communicate and share information for each part via cloud as a collector of data using computing intelligence.

The mentioned facts make the demand for research and development of new modern methods with are in line with the vision of Industry 4.0. One of these methods is also recognition and evaluation of welds [3].

The quality of weld joint must be evaluated during the whole welding process not only by testing of finished joint or construction. In terms of construction quality, weld joints are the most dangerous place in construction. This is the reason why the quality of weld joints is very important. [3]

The basic parts of the weld joint can be seen in Fig. 1:

1. Fundamental material

2. Boundary of weld

3. Weld reinforcement
4. Groove face

5. Root of weld

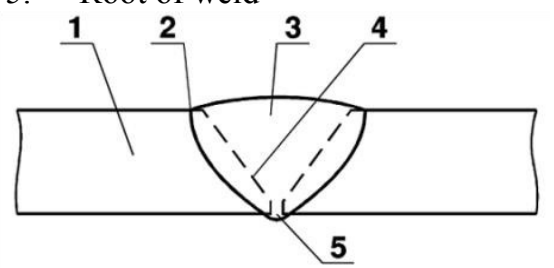

Fig. 1 Illustrated main terms of weld joint [3].

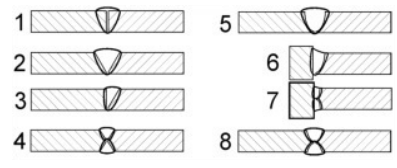

Fig. 2 Some butt weld types [3]

A. Selected weld types

Some types of butt welds are illustrated Fig. 2 , namely:
1. square
2. single- $\mathrm{V}$
3. single bevel
4. double-V
5. single-U
6. single-J
7. double $\mathrm{J}$
8. double side U-weld

\section{B. Defections of weld}

During all welding processes, there are many problems and most of them refer to non-integrity. The main quality of weld criteria is shape, integrity and required physical and chemical features. [3]

Butt weld defects are divided generally to internal and surface. To surface defects belongs: excess weld metal (Fig. 3), overlap, imperfect shape, root concavity, excessive meltthrough, undercuts (Fig. 4), poor restart (Fig. 3), cracks and spatter (Fig. 5) incomplete root penetration.

Testing using visual is non-destructive weld joints testing. Visual examination is performed at first before the others. Visual examination often reveals the most of the defects so the other time-consuming and expensive examinations are not done.

The visual examination focuses on the overall condition of the weld. The examination is performed by worker's eyes. The distance of examination should be $600 \mathrm{~mm}$ above surface and angle should not be lower than $30^{\circ}$. [3]

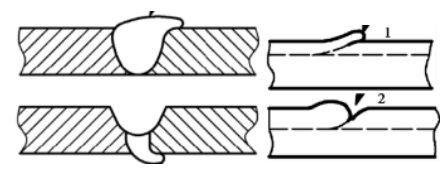

Fig. 3 Excess metal of weld (left) and poor restart (right) [2]

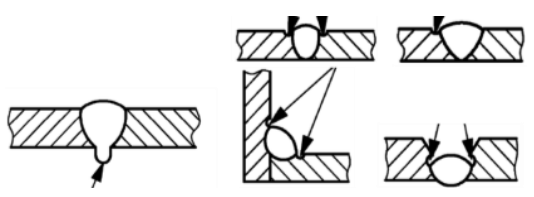

Fig. 4 Excessive melt-through (left) and undercut (right and middle) [2] 


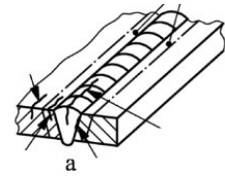

Fig. 5 Examples of cracks [2]
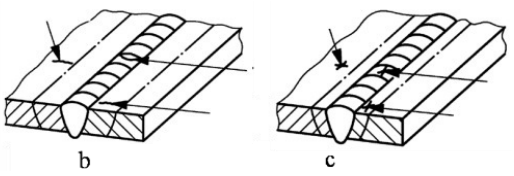

\section{CONCEPTION OF SYSTEM FOR WELD RECOGNITION AND DIAGNOSTIC}

Based on analysis of methods for weld evaluation, computer vision and industrial trends, technology there were proposed system for weld recognition and evaluation. In Fig. 6 is shown the scheme. Main parts of the system are singleboard computer Raspbery Pi 2 Model B, camera IDS uEye $\mathrm{XS}$ and web service Azure Machine Learning (Haffner, 2016).

Computing and communication kernel of this system is single-board computer Raspberry Pi 2 Model 2. Computer provides communication with camera which is mounted to laboratory preparation and takes images of weld samples. Obtained image data are processed and result is weld segmentation. Segmentation is used for computing of invariant image moment and then saved to text file. Text file is read by communication programme and sent to web service. Web service based on input data provide classification based on artificial computing intelligence and results are send back to communication programme.

Each parts of system are realised using low-cost components. Some of components are not suitable for industrial environment, however, for research and evaluation of proposed new modern method are. Using of low-cost components enables to utilize proposed system also for pedagogical purposes or modern ways for teaching of weld specialist.

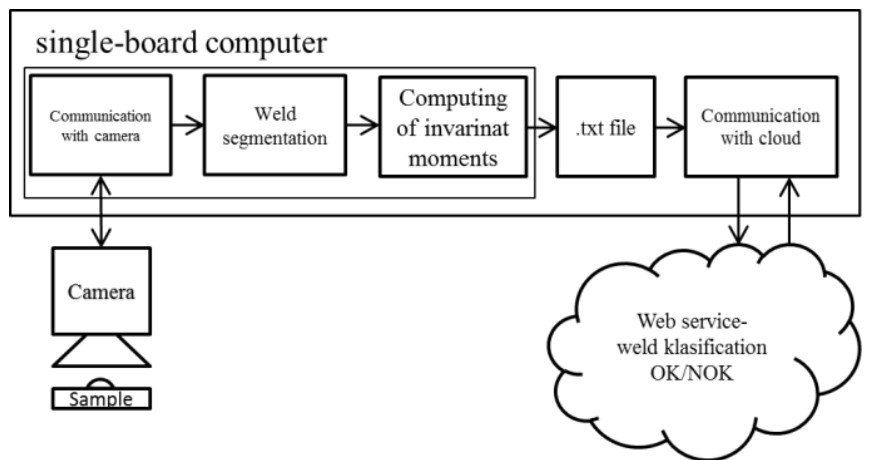

Fig. 6 Conception scheme for system for weld recognition and diagnostic

\section{COMPUTING TIME ANALYSIS}

Based on proposal method for weld recognition and evaluation, we made simple computing time comparison of chosen computer vision algorithms. As input image, we used image of laboratory made weld (Fig. 7 left). One tested algorithm is algorithm made in our work (Haffner \& Duchoň, 2014). This algorithm makes an environment map for mobile robot. In the next part of this section we will describe tested algorithms in detail.

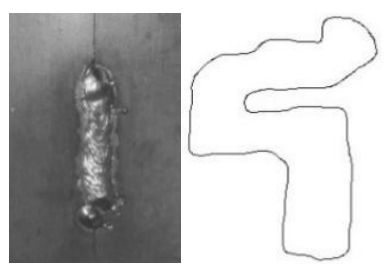

Fig. 7 Laboratory made weld (left) and result of algorithm for map making.

\section{A Tested algorithms}

All tested algorithms are realized in computer vision library OpenCV.

Used threshold algorithm uses Gauss method and block size for neighborhood 15 .

Opening algorithm is realized as erode and then dilate. The structuring element has ellipse shape and size $5 \times 5$.

Blur is realized as Gauss blur with kernel size $11 \times 11$.

Algorithm for map making will be described briefly. The laser scanner data are loaded from text file. Based on measured data the coordinates are computed and plotted in image. After plotting the map is thresholded. Then multiple time blur and threshold is used. After this Zhang-Suen skeletonizing is used. The result of this algorithm is in Fig. 7 right.

\section{B Computing time measurement}

Tested algorithms was compiled and measured on Raspberry Pi, Banana Pi, Raspberry PI2 and personal computer with CPU Intel Core i7 $9302.80 \mathrm{GHz}$. Result of simple time measurement we can see in Table 1. Based on result of the measurement, the Banana $\mathrm{Pi}$ is the fastest among embedded computers even when it's CPU has 2 cores and Raspberry Pi2 CPU has 4 cores. Better results from Raspberry PI2 can be expected using optimized algorithms for multi-core CPU.

Table 1

\begin{tabular}{|c|c|c|c|c|c|c|c|c|c|c|c|c|c|c|c|c|}
\hline Algorithm & \multicolumn{4}{|c|}{ Adaptive thresholding } & \multicolumn{4}{|c|}{ Gauss blur } & \multicolumn{4}{|c|}{ Map } & \multicolumn{4}{|c|}{ Opening } \\
\hline Device & Raspb. PI & Banana PI & Rasp. PI 2 & PC & Raspb. PI & Banana PI & Rasp. PI 2 & PC & Raspb. PI & Banana PI & Rasp. PI 2 & PC & Raspb.PI & Banana PI & Rasp. PI 2 & PC \\
\hline \multirow{5}{*}{ Measur.[ms] } & 769 & 310 & 445 & 14 & 1600 & 568 & 884 & 19 & 6125 & 3343 & 5123 & 333 & 947 & 1457 & 1424 & 9 \\
\hline & 750 & 309 & 446 & 14 & 1533 & 569 & 855 & 19 & 6067 & 3451 & 5224 & 302 & 958 & 1456 & 1420 & 10 \\
\hline & 780 & 309 & 449 & 15 & 1518 & 577 & 851 & 20 & 6264 & 3358 & 5224 & 301 & 943 & 1456 & 1444 & 9 \\
\hline & 763 & 304 & 441 & 16 & 1517 & 570 & 853 & 19 & 6058 & 3365 & 5067 & 303 & 968 & 1460 & 1421 & 10 \\
\hline & 760 & 311 & 457 & 14 & 1538 & 593 & 857 & 19 & 6073 & 3439 & 5059 & 302 & 969 & 1456 & 1439 & 10 \\
\hline
\end{tabular}




\section{VISUAL SYSTEM}

Industrial camera IDS uEye XS for image acquisition was used. Advantage of this camera are measurements, USB 2.0 connection, plenty of integrated functions and SDK for embedded operating systems and processors with ARMv7 Cortex.

\section{A Industrial camera IDS uEye XS}

Camera uEye XS is USB 2.0 industrial camera by company IDS Imaging Development Systems. It has 5.04 Mpix CMOS sensor by company ON Semiconductor with pixel size $1,4 \mu \mathrm{m}$. Supplied complex software package enables to integrate camera to application using standard interface as DirectShow, ActiveX, GenICam or directly by using uEye API.

\section{B Communication with Raspberry Pi 2}

For camera communication with single-board computer Raspberry Pi 2 was used SDK IDS Software Suite and operating system Raspbian based on Linux. SDK obtain libraries and header files and enable using function which operate with camera. The basic principle is shown in Fig. 9. In general, image from camera is loaded in a loop, until the key is pressed. To the image is plotted rectangle which represents region of interest in image (Fig. 8). The weld sample should be located to this area.

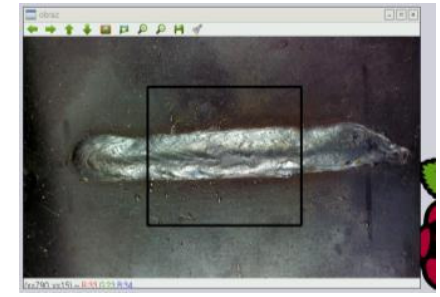

Fig. 8 Window with captured image of weld in ROI

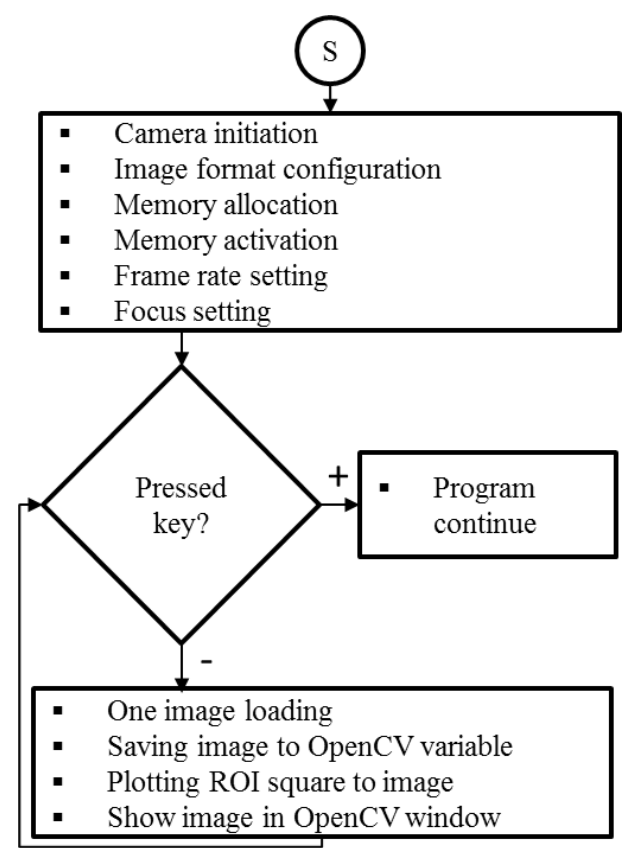

Fig. 9 Camera communication scheme

\section{Laboratory experiment}

For the tasks of image acquisition and processing there was needed to propose the preparation where will be camera mounted and take images of laboratory prepared weld samples under the artificial light. The experiment (Fig. 10) was constructed using low-cost components as building kit Merkur and ligh source (12V LED spot lights).

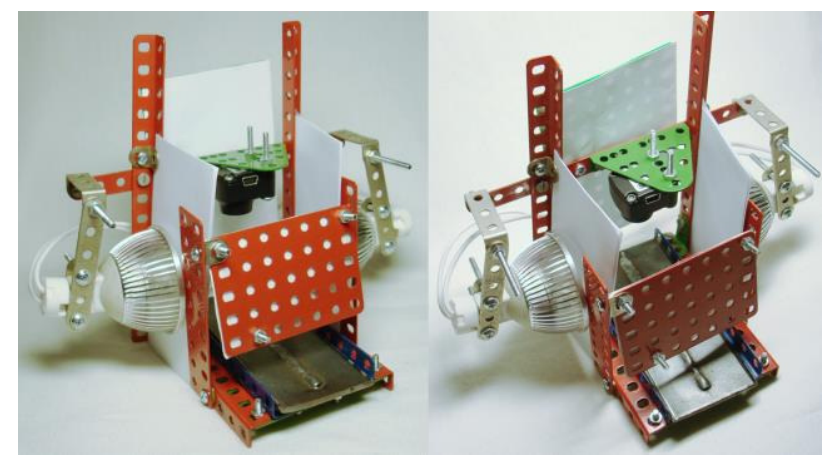

Fig. 10 Laboratory experiment

\section{WELD SEGMENTATION}

The weld segmentation method is based on namely local entropy of image which works with a statistical measure of randomness. Using this method comes from idea, that the surface around weld is flat and monotone however welded area has textured (random) surface.

\section{A Experiment}

We used Matlab Image processing toolbox and laboratory made weld samples for this experiment. The image (Fig.11 a) is filtered at first by median filter with 5-by5 neighborhood around the corresponding pixel. After median filtering the entropy filter is applied. The neighbourhood for filtering is 15-by-15. Resulting image (Fig.11 b) is threshelded with threshold 165 . The results of thresholding we can see in Fig. 11 c. As we can see, the thresholding did not give ideal results. Around weld mask are several blobs. Blobs are removed using the Matlab function bwareaopen. The basic steps of this algorithms are: determining the connected components, computing the area of each component and removing small objects. In our experiment we remove objects smaller than 2000 pixels. The result of removing small objects we can see in Fig.11 d.

The mask contains unfilled holes (Fig12a). The wholes are filled using binary closing with disk structural element with size 10 (Fig.12b). In the Fig.12c we can see the result of weld segmentation and in the Fig.12d we can see corresponding edges of segmentation.

Also the others weld samples was tested with the same parameters. The results we can see in Fig. 13 and 14 as a) original image, b) entropy, c) mask, d) highlighted edges of segmented weld. 


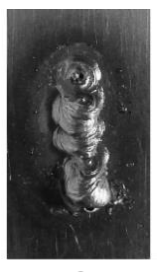

a

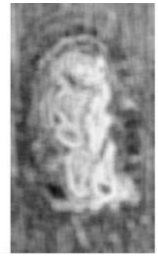

b

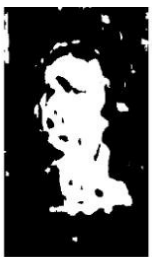

c

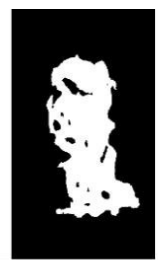

d
Fig. 11 a) original image of weld, b) entropy filtered image, c) thresholded entropy image, d) removed blobs

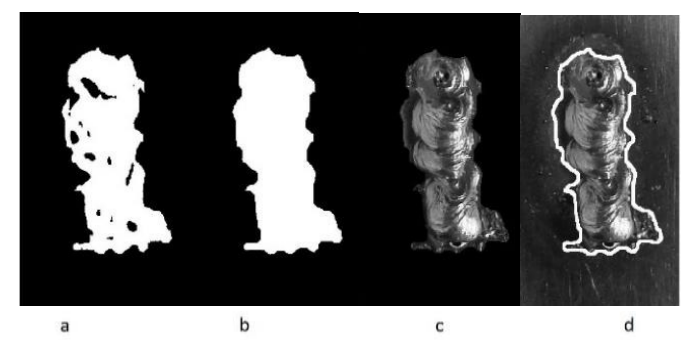

Fig. 12 a) original mask, b) filled mask, c) segmented weld, d) highlighted edges of segmented weld

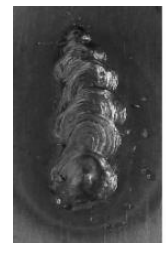

a

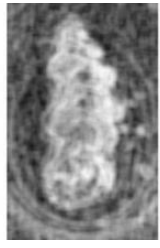

b

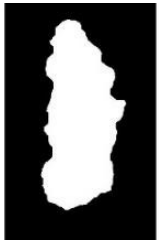

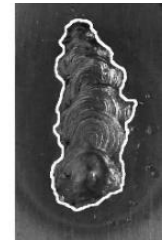

d
Fig. 13 Weld sample 1

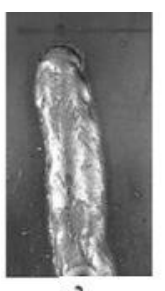

a

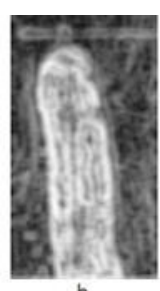

$2^{b}$

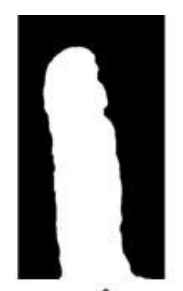

c

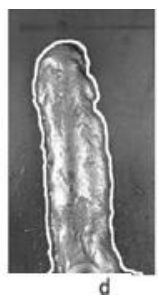

Fig. 14 Weld sample 2

\section{B Algorithm implementation}

Proposed algorithm for weld segmentation needs to be converted to $\mathrm{C}$ code, which is able to implement to singleboard computer Raspberry Pi 2. This can be done using MATALB Coder.

The proposed algorithm had to be adjusted. MATLAB Coder do not support al MATLAB functions and some function had to be replaced by functions from other toolbox. Function bwareaopen from Image Processing Toolbox had to be replaced by vision.BlobAnalysys from Computer Vision Toolbox. The function for entropy computing is not supported at all. This was the reason, why algorithm had to be adjusted.

Tasks for thresholding, blob removal and masking was moved to separate function. The input parameter is image entropy and output is segmented image. Computing of image entropy is not done using MATLAB but programme realization using OpenCV. The resulting image is transpositioned because of different data representation in
MATALB and OpenCV. After transpositin the image is converted to array and this array is input parameter for function generated from MATALB Coder. This proces is illustrated in Fig. 15.

\begin{tabular}{|c|c|c|c|c|c|}
\hline $\begin{array}{l}\text { grayscale } \\
\text { image }\end{array}$ & $\rightarrow \begin{array}{c}\text { entropy } \\
\text { computing- } \\
\text { OpenCV }\end{array}$ & transposition & $\begin{array}{c}\text { Conversion to } \\
\text { array }\end{array}$ & $\begin{array}{l}\text { segmentation- } \\
\text { code from } \\
\text { MATALB }\end{array}$ & $\begin{array}{c}\text { segmented } \\
\text { image }\end{array}$ \\
\hline
\end{tabular}

Fig. 15 Algorithm implementation scheme

Computing time of implemented code on Raspberry Pi2 (in table as RPi2) was compared with algorithm in MATLAB. Compared was the whole segmentation algorithm, generated function texture_seg2 and entropy computing. Measurement of MATLAB algorithm was done on computer Intel Core i7-3610QM 2.3GHz. in Table 2 we can see the time comparison. The computing time 2,2 seconds is for solved problem satisfying.

Table 2

\begin{tabular}{|c|r|r|r|r|r|r|}
\hline & \multicolumn{2}{|c|}{ segmentation } & \multicolumn{2}{c|}{ texture_seg2 } & \multicolumn{2}{c|}{ entropy } \\
\hline device & RPi 2 & MATLAB & RPi 2 & MATLAB & RPi 2 & MATLAB \\
\hline \multirow{4}{*}{ time[s] $]$} & 2,271 & 0,430 & 0,924 & 0,056 & 1,353 & 0,302 \\
\cline { 2 - 7 } & 2,259 & 0,432 & 0,912 & 0,057 & 1,319 & 0,303 \\
\cline { 2 - 7 } & 2,284 & 0,431 & 0,897 & 0,056 & 1,335 & 0,305 \\
\cline { 2 - 7 } & 2,243 & 0,432 & 0,899 & 0,055 & 1,324 & 0,302 \\
\cline { 2 - 7 } & 2,265 & 0,423 & 0,892 & 0,057 & 1,321 & 0,304 \\
\hline average[s] & 2,264 & 0,429 & 0,904 & 0,0562 & 1,330 & 0,303 \\
\hline
\end{tabular}

\section{Weld representation for classification}

The computing intelligence methods which will classify welds need its suitable representation. The features which will represent the weld were set as invariant moment characteristics. Computing of these characteristics are implemented in library OpenCV. The result is 7 numbers (moments), which are saved in text file. The average computing time of the moment on Raspberry Pi 2 is 5,59E-4 seconds.

\section{WELD CLASSIFICATION}

The feature representation of weld as invariant moments can be used for classification. For classification using computing intelligence method Microsoft Azure Machine Learning was chosen.

For training classifier, it is needed training data. It was unable to make thousands of weld samples. Instead of this, the training data was generated from real images of weld by moving weld through the ROI. 6428 training samples was generated from 50 laboratory samples.

Artificial neural network was used for classifying. There were done 6 experiment. For each experiment was set normalizer as Gauss normalizer. Structures of neural networks was default, i.e. one hidden layer, number of input nodes are the same as number of input features (7), nodes hidden layer is set by experimentator and output layer is set by number of classify classes (2). In Table 3 we can see the set parameters for individual layers. 
Table 3

\begin{tabular}{|l|r|r|r|r|r|r|r|}
\hline & $\begin{array}{c}\text { Hidd. } \\
\text { layers }\end{array}$ & $\begin{array}{c}\text { Learn. } \\
\text { tempo }\end{array}$ & $\begin{array}{c}\text { Numb. } \\
\text { of itter. }\end{array}$ & $\begin{array}{c}\text { Init. Learnig } \\
\text { weight }\end{array}$ & $\begin{array}{c}\text { mom- } \\
\text { entum }\end{array}$ & $\begin{array}{c}\text { Mean sqrd. } \\
\text { deviation }\end{array}$ & $\begin{array}{c}\text { Standard } \\
\text { deviation }\end{array}$ \\
\hline ANN 1 & 15 & 0,1 & 100 & 0,1 & 0 & $1,054 \mathrm{E}-04$ & $2,887 \mathrm{E}-03$ \\
\hline ANN 2 & 15 & 0,1 & 500 & 0,1 & 0 & $1,558 \mathrm{E}-06$ & $4,938 \mathrm{E}-05$ \\
\hline ANN 3 & 20 & 0,1 & 500 & 0,1 & 0 & $2,048 \mathrm{E}-06$ & $6,569 \mathrm{E}-05$ \\
\hline ANN 4 & 20 & 0,1 & 300 & 0,1 & 0 & $7,213 \mathrm{E}-06$ & $2,304 \mathrm{E}-04$ \\
\hline ANN 5 & 15 & 0,1 & 1500 & 0,1 & 0 & $1,159 \mathrm{E}-07$ & $3,401 \mathrm{E}-06$ \\
\hline ANN 6 & 20 & 0,1 & 1500 & 0,1 & 0 & $1,052 \mathrm{E}-07$ & $3,069 \mathrm{E}-06$ \\
\hline
\end{tabular}

Results for experiments ANN 2 to 6 can be characterized so, that all classifications were right in case of true positive result with probability range $0.9-1$ and true negative result with probability range $0,1-0$. In this case Metrics as Accuracy, Precision or Recall is 1 . To compare these neural networks, each other we used exact probabilities of each prediction, which can be exported from the Score module. Experiment with ANN 6 had the best results and was used as a classifier.

The ANN6 classifier was than tested with new dataset. Images for this dataset was generated from previous images, but the images were deformed in graphical software. Using this approach, we generated new dataset. The Mean squared deviation was 3,080E-07 and Standard deviation 6,581E-06. Results confirmed proposed method applicability for the problem of weld evaluation.

\section{VIII.VERIFICATION OF IMPLEMENTATION}

After integration of all parts of the system as communication with visual system, weld segmentation and communication with web service it was possible to verify the functionality of the system and computing time. Recognition provides one console application, which starts the camera image acquisition, image is saved after any key is pressed, with image are executed proposed algorithms, the feature representation is send to the web service and it send the results to the program and the results are showed to the console. Application was tested with set of 14 weld samples which was not used for training dataset. In Table 4 we can see the results.

Table 4

\begin{tabular}{|c|c|c|c|c|}
\hline Num. & classify & predicted & probability & time [s] \\
\hline 1 & OK & OK & $1,000000 \mathrm{E}+00$ & 5,31419 \\
\hline 2 & $\mathrm{OK}$ & OK & $9,999996 \mathrm{E}-01$ & 4,85362 \\
\hline 3 & NOK & NOK & 2,787349E-19 & 5,34659 \\
\hline 4 & NOK & NOK & $3,546546 \mathrm{E}-13$ & 5,16983 \\
\hline 5 & OK & OK & 9,999999E-01 & 4,87419 \\
\hline 6 & $\mathrm{OK}$ & $\mathrm{OK}$ & $1,000000 \mathrm{E}+00$ & 5,19455 \\
\hline 7 & OK & OK & $9,999998 \mathrm{E}-01$ & 4,86545 \\
\hline 8 & NOK & NOK & $8,053897 \mathrm{E}-11$ & 5,16845 \\
\hline 9 & NOK & NOK & $2,887203 \mathrm{E}-15$ & 4,96445 \\
\hline 10 & NOK & NOK & $4,755493 \mathrm{E}-32$ & 5,44418 \\
\hline 11 & NOK & NOK & $3,072803 \mathrm{E}-13$ & 5,34558 \\
\hline 12 & NOK & NOK & $2,193890 \mathrm{E}-16$ & 5,10893 \\
\hline 13 & NOK & NOK & $4,700284 \mathrm{E}-12$ & 5,18963 \\
\hline 14 & $\mathrm{NOK}$ & $\mathrm{NOK}$ & $3,459683 \mathrm{E}-09$ & 4,95562 \\
\hline & & & average & 5,128233 \\
\hline
\end{tabular}

\section{CONCLUSION}

The performed analysis of the current state in pattern recognition applications for welding has revealed that there has not been implemented and presented a solution based on low-cost single-board computer using cloud technologies yet. The proposed solution enables to recognize a weld in 5,2 seconds which is satisfactory for 0,5-1minute-long manufacturing tact used in automotive industry, and is suitable even for recognition of larger weld deformations. The used low-cost components are suitable for small and medium-sized companies. The proposed methodology can be effectively used especially in automotive industry.

\section{ACKNOWLEDGMENT}

This work has been supported by the Cultural and Educational Grant Agency of the Ministry of Education, Science, Research and Sport of the Slovak Republic, KEGA 030STU- 4/2015, KEGA 011STU-4/2015 and KEGA 030STU-4/2017, by the Scientific Grant Agency of the Ministry of Education, Science, Research and Sport of the Slovak Republic under the grant VEGA 1/0819/17.

\section{REFERENCES}

[1] Deng, S., LIpei, J., \& Long, X. (2008). Detecting linear features in weld seam images based on beamlet transform. 2008 9th International Conference on Signal Processing (s. 1145-1148). IEEE.

[2] Haffner, O. (2016). Contribution to modern methods. (in slovak). Ph.D. thesis: Slovak University of Technology in Bratislava.

[3] Haffner, O., \& Duchoň, F. (2014). Making a Map for Mobile Robot Using Laser Rangefinder. 23rd International Conference on Robotics in Alpe-Adria-Danube Region. Conference Proceedings. Bratislava: Publishing House of Slovak University of Technology.

[4] Haffner, O., Kučera, E., \& Kozák, Š. (2016). Weld Segmentation for Diagnostic and Evaluation Method. Levoča: 2016 Cybernetics \& Informatics (K\&I), IEEE. doi:10.1109/CYBERI.2016.7438605

[5] Hou, X., \& Liu, H. (2012). Welding Image Edge Detection and Identification Research Based on Canny. 2012 International Conference on Computer Science and Service System.

[6] Kagermann, H. (2013). Recommendations for implementing the strategic initiative INDUSTRIE 4.0. Dostupné na Internete: http://www.acatech.de/fileadmin/user_upload/Baumstruktur_nach_W ebsite/Acatech/root/de/Material_fuer_Sonderseiten/Industrie_4.0/Fina 1_report_Industrie_4.0_accessible.pdf

[7] Liao, Z., \& Sun, J. (2013). Image segmentation in weld defect detection based on modified background subtraction. 2013 6th International Congress on Image and Signal Processing (CISP). IEEE.

[8] Marônek, M., \& Bárta, J. (2008). Multimediálny sprievodca technológiou zvárania (elektr. monografia). Trnava: AlumniPress.

[9] Mař́k, V. (2015). National initiative Industry 4.0. Available on Internet:

http://download.mpo.cz/get/53723/62020/640376/priloha001.pdf

[10] Shen, Z., \& Sun, J. (2013). Welding seam defect detection for canisters based on computer vision. 2013 6th International Congress on Image and Signal Processing (CISP).

[11] Ulrich, K., Koleňák, R., \& Karvanská, S. (2006). Skúšanie zvarových spojov. Bratislava: STU v Bratislave.

[12] Xuming, Z., Zhouping, Y., \& Youlun, Y. (2007). Edge Detection of the Low Contrast Welded Joint Image Corrupted by Noise. 8th International Conference on Electronic Measurement and Instruments. Lab., Cambridge, MA Rep. ARCRL-66-234 (II), 1994, vol. 2. 